\title{
O conceito de integrais nos cursos de Cálculo
}

\author{
José C. Magossi e Antônio C. C. Barros
}

\section{Resumo}

O conceito de integrais, grosso modo, nasceu na Grécia antiga e sofisticou-se após a descoberta do Cálculo por I. Newton e G. Leibniz. Com tais cientistas surge o Teorema Fundamental do Cálculo, que relaciona o conceito de diferenciação com o de integração, e responde, de certa forma, o problema grego da determinação de áreas de regiões não regulares. Quase dois séculos após, A. Cauchy entende que o conceito de integrais deve ser desenvolvido de modo independente do conceito de derivadas. Para isso, cria o conceito de limites. Na sequência, B. Riemann expande os trabalhos de Cauchy e enuncia condições para que uma função seja integrável. As classes de funções integráveis expandem-se novamente com os trabalhos de H. Lebesgue, O. Perron, A. Denjoy, J. Kurzweil e R. Henstock, entre outros. Neste artigo, o objetivo é exibir exemplos de funções integráveis, e não integráveis, de acordo com suas definições. Isso pode facilitar o entendimento das fronteiras do conceito de integração e mostrar aos alunos que resolver integrais não se limita apenas a encontrar primitivas.

Palavras-chave: Integrais; Newton-Leibniz; Cauchy; Riemann; Lebesgue; Kurzweil-Henstock.

\begin{abstract}
The integrals concepts started, roughly speaking, in Ancient Greece, and was later enhanced after the discovery of the calculus by I. Newton and G. Leibniz. These scientists give birth to the Fundamental Theorem of Calculus, which relates the differentiation concept to the integration one, and provides the answer, in a way, to the Greek problems concerning of determining areas of non-regular regions. Nearly two centuries later, A. Cauchy understands that the integrals concept must be developed in an independent way from the concept of derivatives. To this end, the limit concept is developed by Cauchy. In the historical sequence, B. Riemann expands Cauchy's work and enunciates conditions for a function to be integrable. The classes of integrable functions expand once again with the works of H. Lebesgue, O. Perron, A. Denjoy, J. Kurzwell, and R. Henstock, among others. In this article, the aim is to show examples of integrable and non integrable functions, according to their definitions, which will foster the understanding of the boundaries in the integral concept, and also show students that solving integrals is not limited to only determine the primitives.
\end{abstract}

Keywords: Integrals; Newton-Leibniz; Cauchy; Riemann; Lebesgue; Kurzweil-Henstock. 


\section{Introdução}

O conceito de integrais tem uma forte relação com geometria, pois na Grécia antiga, em particular, relacionavam-no à busca por áreas de regiões não regulares. Por isso, é comum limitar o escopo desse conceito ao de área. Com a descoberta do Cálculo, por Isaac Newton (1643-1727) e Gottfried W. Leibniz (1646-1716), essas ideias avançam em sofisticação, e com o Teorema Fundamental do Cálculo dá-se a relação entre diferenciação e integração. Esse teorema abre portas para fornecer uma resposta positiva, por conseguinte, ao problema grego da determinação de um valor para áreas de regiões não regulares. A área de uma região acima do eixo $x$ entre as retas $x=a \mathrm{e}$ $x=b$, sob uma curva contínua determinada por $f(x) \geq 0$, é calculada, em notação atual, como $\int_{a}^{b} f(x) d x=F(b)-F(a)$, desde que $F:[a, b] \rightarrow \mathbb{R}$ e $F^{\prime}(x)=f(x)$ para todo $x \in[a, b]$. Nesse caso, a função $F(x)$ é denominada de função primitiva de $f(x)$. Diz-se ainda que $f(x)$ é uma função primitivável. Não há como negar o impacto que esse teorema acarretou na matemática nos séculos XVIII e XIX. Com uma intuição, magistral, Augustin Louis Cauchy (1789-1857), em seu Cours d'analyse de l'ecole royale polytechinque. $1^{\text {re }}$ partie: analyse algébrique, de 1821, prepara os fundamentos do rigor em matemática, e, em seguida, em seu Résume des leçons donnés a l'ecole royale polytechnique sur le calcul infinitesimal, de 1823, expõe a definição de integral como limites de somas e com demonstrações rigorosas [17]. Por um lado, com o operador limites, Cauchy coloca precisão no conceito de derivadas, por outro, ele trabalha no sentido de eliminar "as derivadas" da definição do conceito de integrais. Cauchy desenvolve o conceito de integrais, para todo $x \in[a, b]$, em termos do limite de somas $S=\sum_{i=1}^{n} f\left(x_{i-1}\right)\left(x_{i}-x_{i-1}\right)$ em que $a=x_{0}<x_{1}<\ldots<x_{n}=b$ é uma partição do intervalo $[a, b]$, para funções $f(x)$ adequadas, contínuas, de números reais. É clássico na história da matemática que o conceito de funções, na época de Cauchy, não estava em patamares matemáticos sólidos, fazendo com que ele cometesse alguns erros ao lidar com o conceito de continuidade, principalmente com o de continuidade uniforme ([20], p9). Mas é notório que a matemática mudou a partir da época de Cauchy e de suas definições de limites e integrais. Cauchy, que nasceu em 1789, ano da Revolução Francesa, também proporcionou, ele próprio, uma revolução, mas científica [17], conforme as ideias de Thomas Kuhn [25]. É um problema investigativo indicar quais foram as razões que levaram Cauchy a trabalhar com seus limites e integrais, mas sabe-se, além disso, que a teoria de integração avançou graças ao desenvolvimento, em paralelo, do conceito de funções. Logo após o surgimento das integrais de Newton e Leibniz, a busca por funções que tenham primitivas incentivou o surgimento de técnicas, algoritmos, diga-se de passagem, que facilitassem essa busca. Como o conceito de funções não se encontrava ainda em bases sólidas ocorria, entre outros problemas, a falta de entendimento do porquê algumas funções não tinham primitivas.

A procura por funçôes primitivas é essencialmente uma busca por um algoritmo que determine quais funções elementares são primitivas. (Grosso modo, uma função elementar é qualquer função que pode ser obtida pressionando-se uma sequência finita de botôes numa calculadora científica.) Tal algoritmo, baseado em investigações passadas feitas por Liouville nos anos de 1830, foi obtido por Risch em 1960, e versões aprimoradas dele são empregadas em pacotes algébricos computacionais atuais. Como resultado pode-se afirmar categoricamente que não existe uma primitiva elementar para $\exp \left(-x^{2}\right)$, mas a teoria é sutil: por exemplo $\sqrt{\operatorname{tg} x}$ e $x \operatorname{sen} x$ ambas têm tais primitivas mas $\sqrt{\operatorname{sen} x}$ e $x \operatorname{tg} x$ não têm (...). (Tradução dos autores) ([23], pp13-14) 
A instabilidade do conceito de funções ganha força com os trabalhos de Jean B. J. Fourier (17681830), matemático contemporâneo de Cauchy, que em 1807 deposita, na hoje conhecida como Academia de Paris, um trabalho sobre condução de calor em uma barra metálica com o objetivo de concorrer ao prêmio proposto pela Academia. Fourier expõe uma solução formal do problema. Os conceitos de convergência, funções, continuidade, séries de funções etc., e também o conceito de integrais, não se encontravam bem fundamentados matematicamente na época de Fourier, e, desse modo, sua solução foi questionada pelos jurados do concurso. Mesmo assim, seu trabalho impactou significativamente na matemática, seja no desenvolvimento do conceito de funções, seja no desenvolvimento do conceito de integrais ou seja nos desenvolvimentos de propriedades de convergência [37]. Ao trabalhar na elucidação dos problemas deixados por Fourier, em síntese, - quais funções podem ser escritas como séries infinitas de senos e cossenos-, Georg F. B. Riemann (1826-1866) volta-se para o conceito de integrais e levanta o seguinte questionamento: Para quais funções tem-se $\int_{a}^{b} f(x) d x$ ? B. Riemann desenvolve critérios para indicar se uma função é ou não integrável. Em sua época, ele expande a classe de funções integráveis no sentido de Cauchy e vai além das funções integráveis que sejam contínuas ou descontínuas em um conjunto finito de pontos, tal como exposto por Cauchy.

Riemann mostra que há funções integráveis com número infinito de pontos de descontinuidade ${ }^{1}$. Com Riemann tem-se a primeira caracterização sistemática de funções integráveis $[19,20,24]$.

Como em todo desenvolvimento matemático é comum ocorrer "deficiências" que indicam que ajustes precisam ser realizados, o mesmo se deu com as integrais de Riemann. A classe de funções integráveis é pequena, nem toda função Riemann integrável tem primitiva, há funções que têm primitivas mas não são Riemann integráveis etc. [3].

Além de Riemann, outros matemáticos preocuparam-se com os problemas deixados por Fourier e também pela solução das deficiências presentes nas integrais de Riemann. Em particular, o francês Henri Leon Lebesgue (1875-1941), publica no início do século XX, alguns trabalhos com o intuito de expandir a classe das funções integráveis e, principalmente, com o objetivo de "integrar todas as funções primitiváveis". A nova definição de integral dada por Lebesgue remove a restrição, nas integrais de Riemann, de que o integrando seja limitado e que o intervalo de integração seja compacto. Junto com a definição de Lebesgue vem uma Teoria da Medida para sustentar seu conceito de integral $[4,5,23]$. No entanto, mesmo com a definição de Lebesgue, que aumentou significativamente a classe de funções integráveis, ainda assim algumas deficiências permaneciam, como a ideia de que cada função primitivável seja integrável. Uma outra deficiência, digamos pedagógica, se apresenta na integrais de Lebesgue: os pré-requisitos para o bom entendimento de sua teoria da medida e integração acabam por ser complicados demais aos alunos iniciantes. Um ponto positivo é que, com base na integrais de Lebesgue, os problemas deixados por Fourier são resolvidos. Mais ainda, as integrais de Lebesgue auxiliam no desenvolvimento de ferramentas matemáticas em áreas tais como mecânica estatística, teoria de probabilidades etc.

Com foco na ideia de integrar todas as funções primitiváveis, Arnauld Denjoy (1884-1974) e Oskar Perron (1880-1975) desenvolvem, de modo independente, no início do século XX, abordagens distintas para novos conceitos de integrais, os quais integram todas as funções que tenham primitivas. As duas abordagens mostram-se equivalentes, ou seja, produzem a mesma classe de funções integráveis. Se as integrais de Lebesgue são consideradas difíceis para alunos iniciantes, as de Denjoy

\footnotetext{
${ }^{1}$ Em linguagem atual diz-se que: Uma função limitada $f:[a, b] \rightarrow \mathbb{R}$ é Riemann integrável se e somente se ela é contínua em quase todo ponto em $[a, b]$, ([3], p221).
} 
e Perron são consideravelmente mais difíceis ([2], pxi). No entanto, a simplicidade da definição de integrais ao estilo de Riemann (e Cauchy) deixam de existir ao tratar-se com integrais de Lebesgue, de Denjoy e de Perron, haja vista exigirem conhecimentos matemáticos avançados para seu entendimento. Por volta de 1960, J. Kurzweil (1926-) e R. Henstock (1923-2007) propõem uma nova abordagem sobre integrais, fundamentada nas ideias simples de somas de Riemann [21, 26]. Com isso obtém-se uma classe de funções integráveis que engloba todas as anteriores, e com uma exposição que pode ser exposta aos alunos iniciantes [2, 3, 16, 27]. Além disso, as integrais de Kurzweil-Henstock são equivalentes, em termos de classe de funções integráveis, às de Denjoy e de Perron. As integrais de Kurzweil-Henstock, ou integrais de Riemann generalizadas, ou ainda integrais de calibres, $[2,16]$, ganham adeptos na comunidade matemática ao ponto de haver listas de discussões na internet onde advogam-se em prol da utilização dessas integrais em cursos de Cálculo, em lugar das clássicas integrais de Riemann. À parte de advogar, neste artigo, em prol de uma ou de outra abordagem sobre integrais, é possível encontrar discussões interessantes sobre esse assunto no endereço eletrônico https://math.vanderbilt.edu/schectex/ccc/gauge/ ${ }^{2}$.

Diante da breve exposição nas linhas acima, é possível inferir que o assunto teoria da integração ainda está em pleno desenvolvimento. Há livros destinados aos alunos de graduação que tratam das integrais de Riemann em paralelo às integrais de Riemann generalizadas [3, 27]. Com o avanço das publicações desses recentes desenvolvimentos em integrais, é interessante que sejam escritos textos destinados aos alunos iniciantes no sentido de clarear o cenário em que as integrais estejam presentes. Por exemplo, em livros antigos encontram-se frases do tipo: "a função $f(x)$ é integrável". Já em livros modernos e em publicações atuais encontram-se frases do tipo: "a função $f(x)$ é Riemann-integrável," "a função $f(x)$ é Lebesgue-integrável," "a função $f(x)$ é NewtonLeibniz integrável," "a função $f(x)$ é Kurzweil-Henstock-integrável" etc. É importante que haja classificações, um quadro comparativo com relação às funções, para saber se determinada função é ou não integrável com respeito a determinada definição de integral. Ou seja, ao se ler em um livro que $f$ é integrável, se quer dizer então que $f$ é integrável no sentido de Riemann? Ou no sentido de Cauchy? Ou no sentido de Lebesgue? Ou no sentido de Darboux? Ou ainda no sentido de Kurzweil-Henstock? É evidente que algumas classes são equivalentes, mas um esclarecimento pode facilitar o caminhar do leitor. Urge que os alunos visualizem que resolver integrais não se resume na busca por funções que tenham primitivas ao estilo das integrais de Newton e Leibniz. Estima-se que ao exibir uma tabela comparativa, via exemplos ilustrativos, de funções integráveis de acordo com suas definições, seja possível avançar na compreensão e entendimento do conceito de integrais. O objetivo neste texto é tanto didático quanto matemático. A parte didática, tem-se em conta, vai auxiliar na compreensão do conceito de integrais em suas várias facetas. A parte matemática indica uma compilação de diferentes conceitos de integral e sua relação com a referida classe de funções obtida. Do mesmo modo que pode ser útil ao pesquisador em matemática, ou professor, por exemplo, saber de exemplos de funções que são Riemann integráveis mas não são Newton integráveis, pode ser igualmente útil ao aluno perceber que resolver integrais não se resume simplesmente a encontrar primitivas. Assim, objetiva-se exibir, sem pretensões de completude, exemplos e contraexemplos de funções integráveis, e não integráveis, de acordo como o conceito de integral tenha sido definido. É evidente que a face generalista da matemática incide sobre o conceito de integral [16] de modo a torná-lo, com os desenvolvimentos subsequentes, o mais extensivo possível e com impacto em diversas áreas da matemática. É clássico na matemática que a teoria moderna de integração é um dos pilares que sustentam a análise matemática, e que uma grande parte da matemática vale-se dos conceitos advindos da análise matemática [2]. Desse

${ }^{2}$ Acessado em 05 de dezembro de 2019 às 12 h34min. 
modo, o conhecimento do conceito de integrais torna-se útil para a ciência em geral e, mais ainda, alterações nesses conceitos podem impactar em diversas áreas. A teoria da integração é ampla e com muitas ramificações. Desse modo, neste texto, opta-se pela abordagem das classes de funções integráveis que, entende-se, acarretaram alterações significativas. São elas, as classes de NewtonLeibniz, Cauchy, Riemann, Lebesgue e Kurzweil-Henstock.

\section{Funções e Integrais}

Um dos motivos que levam à expansão das classes de funções integráveis é devido, às vezes, às limitações presentes nas definições vigentes. Com os trabalhos de Cauchy pode-se citar a preocupação com a pedagogia em seus cursos, o estabelecimento de rigor na matemática e a independência da definição de integrais do conceito de derivadas. Com Riemann houve um primeiro tratamento sistemático sobre o conceito de integrais e o de classe de funções integráveis, além, é claro, da expansão da classe de funções integráveis em relação às de Cauchy, pois Riemann entendeu que a exigência de continuidade não era essencial ([20], p32). Lebesgue, por sua vez, queria integrar funções que não eram Riemann integráveis, tais como o exemplo de Volterra ([8], p16 e p70). Kurzweil desenvolveu suas integrais com o objetivo de investigar equações diferenciais e Perron queria integrar todas as funções primitiváveis.

Isso pode parecer estranho ao se tratar de matemática, haja vista ser conhecida como uma ciência cumulativa. No entanto, o fato de a matemática poder ser vista como sendo composta de estruturas e objetos, suas múltiplas leituras científicas permitem alterações em suas estruturas e a criação de novos conceitos. Um exemplo clássico básico é o teorema de Pitágoras e suas inúmeras demonstrações [14]. Segundo as ideias de Felix Klein, fazer matemática também é reescrevê-la sob uma outra forma, isto é, resolver o mesmo problema, mas de uma forma diferente [29, 30]. Seguindo essa linha de pensamento, de Felix Klein, algo semelhante ocorre com o conceito de integral ${ }^{3}$. Enquanto que o conceito de derivada encontra-se nos livros de Cálculo quase da mesma forma em que foi enunciado por I. Newton e G. Leibniz no século XVII, o mesmo não se dá com o conceito de integral, o qual começou a ser esboçado na Grécia antiga, por Arquimedes, passou por I. Newton (1642-1727) e G. W. Leibniz (1646-1716), A. L. Cauchy (1789-1857), J.G. Darboux (1842-1917), G. F. B. Riemann (1826-1866), T. J. Stieltjes (1856-1894), H. Lebesgue (1875-1941), E. J. McShane (1904-1989), A. Denjoy (1884-1974), O. Perron (1880-1975), R. Henstock (1923-2007) e J. Kurzweil (1926-), para citar alguns ${ }^{4}$. Cada nova definição de integral implica alguma inovação que pode gerar uma nova classe ou se revelar equivalente às anteriores.

Com foco nos processos de transformação da matemática, estima-se que o esclarecimento das classes de funções integráveis de acordo com suas definições pode acarretar melhorias na interação e compreensão de conceitos. Espera-se que perguntas como $O$ que é uma integral? possam ser respondidas com consistência por alunos iniciantes, leitores etc., cujas respostas não sejam simplesmente: É uma função que tem uma primitiva.

\footnotetext{
${ }^{3}$ Nota-se que o conceito de funções, importante no desenvolvimento das teorias de integração, também sofreu alterações significativas ao longo da história da matemática [20, 24, 34, 41].

${ }^{4}$ Não se pretende fazer uma descrição exaustiva de todos os matemáticos que de uma forma ou outra indicaram alterações no conceito de integral. Pode-se citar também J.C. Burkill (1900-1993), Giulio Ascoli (1843-1896), Henry J. S. Smith (1826-1883), Karl J. Thomae (1840-1921), S. Pollard (1894-1945), B.C. Getchell, W. H. Young (18631942), P.J. Daniel (1889-1946) etc. [8, 21, 33].
} 
A iniciativa de clarear os conceitos e fazer uma releitura apropriada sob a ótica histórica não é isolada, pois muitos livros compartilham dessa preocupação que implica a divulgação, com consistência, de conceitos matemáticos. Um exemplo clássico refere-se à caracterização, em livros de cálculo, dos termos "antiderivada," "primitiva" e "integral indefinida." É comum que sejam tratados como sinônimos, tal como alertado por R. Bartle em seu livro A Modern Theory of Integration ([2], p56). No entanto, caso ocorra, em algum livro de Cálculo, que o conceito de integrais seja exposto apenas sob a ótica de Newton-Leibniz, então, por força do Teorema Fundamental do Cálculo (TFC), a busca por integrais indefinidas, do tipo $F(x)=\int_{a}^{x} f(t) d t$, acaba por ser a busca por antiderivadas $F(x)$ (primitivas) tal que $F^{\prime}(x)=f(x)$ para todo $x \in[a, b]$. Desse modo, é plausível que ocorra uma certa confusão entre antiderivada e integral indefinida. Por conta disso, em alguns livros comentários são descritos com fins de clarear a diferença entre integral indefinida e antiderivada (ou primitiva) ([3], pp216-218). Há também comentários sobre a importância de se explicar a notação, não muito clara, de integral indefinida, comumente escrita como $\int f(x) d x=F(x)+c$ ([11], p189). Isso acaba por gerar dúvidas, entre alunos, sobre o conceito de integrais. Calcular uma integral é encontrar sua antiderivada?, Qual é a relação entre antiderivada e integral indefinida? A notação $\int f(x) d x$ pode indicar simplesmente a classe de funções que tenham primitivas, mas isso não indica necessariamente que seja uma integral indefinida, que pode ser vista como "área sob uma curva". Calcular "integrais" do tipo $\int\left(x^{3}+2\right) d x$ é determinar apenas as classes de antiderivadas, ou primitivas, da função $x^{3}+2$. É certo que existem métodos que indicam "algoritmos" que facilitam a busca por primitivas, tais como "integração" por partes, método da substituição, substituição trigonométrica etc., mas $\int f(x) d x=F(x)+c$ é apenas a indicação de que $F(x)+c$ é uma classe de primitivas (antiderivadas) de $f(x)$. Caso o conceito de integral não seja precisamente definido, corre-se o risco de se confundir o cálculo de integrais com a busca por antiderivadas. $\mathrm{O}$ TFC que, por um lado, é dito Fundamental devido a sua importância na Análise Matemática [6], por outro, pode induzir ao leitor menos atento, o pensamento de que cálculo de integrais resume-se à busca por primitivas. A força do TFC e das integrais, no sentido de Newton-Leibniz, fortalece essa crença. O estudo de integral em Cauchy indica um procedimento construtivo (algorítmico) voltado ao cálculo de integrais e não simplesmente um procedimento descritivo onde se deve "adivinhar" a resposta, ou seja, determinar alguma função primitiva. Integrais de Newton-Leibniz são descritivas, enquanto que integrais de Riemann são construtivas ([40], p175).

Esclarecimentos desse tipo são importantes pois eliminam inconsistências advindas do processo histórico e das transformações matemáticas ocorridas ao longo de anos. Por exemplo, as seções sobre integrais, sejam em livros ou artigos, se o objetivo é didático, poderiam começar, como estratégia pedagógica, pela explicação das integrais de Newton e Leibniz (descritivas) e seguir com as de Cauchy e Riemann (construtivas).

Como um outro exemplo sobre a importância do esclarecimento dos conceitos, dos processos de transformação em matemática, pode-se citar a confusão que se faz ao escrever sobre o tema integrais de Riemann e exibir a definição de integrais de Darboux. Deve-se ter cuidados para não misturar classes equivalentes de funções integráveis, como as de Riemann e Darboux, por exemplo, com suas definições de integrais. Nesse caso, a integral de Riemann tem uma definição distinta da definição de integral de Darboux. Riemann utiliza uma extensão mais aprimorada das somas de Cauchy, enquanto que Darboux utiliza de somas com supremo e ínfimo em sua definição. Gaston Darboux (1842-1917), ao traduzir, em 1873, o trabalho de Riemann para a língua francesa, fez inovações na definição de integral e lançou mão das propriedades de completude do sistema de números reais (supremo, ínfimo, axiomas de completude etc.) para elaborar sua nova definição ([3], p225). Assim, em 1875 ele publica o artigo Mémorie sur la théorie des fonctions discontinues 
[13] e expõe um novo método para identificar funções integráveis, diferente, mas equivalente à classe de funções integráveis de Riemann ([20], p27), ([3], pp225-232). Por isso, entende-se, com base nos exemplos acima, que a identificação de conceitos matemáticos em consonância com a gênese de seu surgimento, [36], favorece o entendimento dos processos de interação e transformação em matemática.

\subsection{Notação para funções integráveis}

É interessante observar como as notações matemáticas têm evoluído ao longo do tempo. No caso de integrais, há alterações desde os tempos de Leibniz até os dias de hoje. Constitui-se também como propósito neste artigo o alerta sobre a importância de uma notação clara para funções integráveis. Assim, diferente da notação advinda dos tempos de Newton e Leibniz, em tempos atuais o conceito de integral está relacionado à notação de integral definida $\int_{a}^{b} f(x) d x$. Leibniz introduziu, em 1686, o símbolo $\mathcal{S}$ estendido, para denotar o símbolo $\int$, utilizado nos dias de hoje. Já o termo "integral" foi introduzido por Johann Bernoulli e foi publicado, em 1690, por seu irmão Jacob Bernoulli ([19], p107). No livro de Florian Cajori há comentários sobre as notações utilizadas para o símbolo de integral e mostra que a de Fourier é utilizada até hoje ([9], pp242-254, Vol. II). O trabalho de J. B. J. Fourier foi publicado em 1822 [15], e, nele, a notação $\int_{a}^{b}$ é exposta, tal como na citação abaixo:

Nós designamos em geral pelo sinal $\int_{a}^{b}$ a integral que começa quando a variável equivale $a$ a, e que está completo quando a variável equivale a b; (Tradução dos autores) ([15], p252, seção 231.)

O trabalho de Riemann sobre representação de funções por séries trigonométricas, publicado em 1854, no qual ele desenvolve suas integrais, é inspirado na busca de uma solução para os problemas deixados por Fourier [31]. Como consequência dessa notação para integrais, tem-se, hoje em dia, a notação para integral indefinida, tal como em [3],

$$
F(x)=\int_{a}^{x} f(t) d t .
$$

Neste texto, dizer que "uma função $f$ é integrável" num intervalo $[a, b]$ é inseri-la em uma das 5 classes: Newton-Leibniz $(\mathcal{N} \mathcal{L}[a, b])$, Cauchy $(\mathcal{C}[a, b])$, Riemann $(\mathcal{R}[a, b])$, Lebesgue $(\mathcal{L}[a, b])$ ou Kurzweil-Henstock $(\mathcal{K} \mathcal{H}[a, b])$.

A exibição de exemplos é um dos objetivos no presente artigo. Não há como negar que os exemplos matemáticos funcionam como divisores de águas, pontos de transformação na matemática. Desse modo, nesse artigo, exibem-se alguns, não exaustivos, exemplos de funções que indicam os limites das classes de funções integráveis. Como é o caso de Vito Volterra (1860-1940) que em 1881 exibiu um exemplo de uma função $F$ diferenciável cuja derivada $F^{\prime}$ é limitada mas não é Riemann integrável [38]. Problemas como esse, entre outros, motivaram Lebesgue a desenvolver um novo conceito de integrais, fundamentado num novo conceito, o de medida.

No que se segue expõe-se, de modo sucinto, para uniformizar o contexto, as definições de integrais relacionadas às cinco classes de integrais discutidas anteriormente, integrais de Newton-Leibniz, 
integrais de Cauchy, integrais de Riemann, integrais de Lebesgue e integrais de Kurzweil-Henstock. As notações e símbolos utilizados são atuais, e seguem àquelas presentes no livro de R. Bartle e D. Sherbert [3].

O objetivo é mostrar que, a partir de alterações no conceito de integral, novas classes, mais abrangentes, são criadas. Mais ainda, procura-se enfatizar que essas alterações são, às vezes, muito sutis, imperceptíveis se não for realizada uma leitura cuidadosa.

\subsection{Integrais de Newton-Leibniz}

A ideia de integral, como é clássico na literatura, e já dito anteriormente, é investigada desde a Grécia antiga, cujo objetivo inicial era o de obter um número associado à área de uma região no plano, ou o volume de uma região no espaço etc. Inúmeras técnicas foram desenvolvidas para esse fim. O surgimento do Cálculo, de certa forma, corresponde ao surgimento do conceito de derivada e, paralelamente, e o de integral.

No entanto, no decorrer desses acontecimentos, Newton e Leibniz fazem uma descoberta interessante, qual seja, que a obtenção da área sob uma curva pode ser obtida com base no processo de antiderivação. Desse modo, surge a clássica fórmula ${ }^{5}$, exposta na definição seguinte, conhecida como integral de Newton-Leibniz, que se refere também ao Teorema Fundamental do Cálculo TFC, pois relaciona o conceito de integral ao conceito de derivadas.

Definição 1. Uma função $f:[a, b] \rightarrow \mathbb{R}$ é dita ser uma função Newton-Leibniz integrável em $[a, b]$ se

$$
\mathcal{N} \mathcal{L} \int_{a}^{b} f(x) d x=F(b)-F(a),
$$

desde que $F:[a, b] \rightarrow \mathbb{R}$ e $F^{\prime}(x)=f(x)$ para todo $x \in[a, b]$.

Neste caso, a função $F$ é chamada de primitiva (ou antiderivada) da função $f$, pois tem-se que

$$
\frac{d}{d x} F(x)=f(x) \quad \text { para todo } x \in[a, b]
$$

Fundamentado nessas ideias, é possível determinar o resultado de integrais por meio de derivadas. $\mathrm{Na}$ maior parte dos textos de Cálculo utilizados em cursos de graduação da área de exatas, a exposição do conceito de integrais ocorre logo após o desenvolvimento do conceito de derivada ${ }^{6}$. Com o TFC alguns problemas podem ser resolvidos, tais como área sob parábolas, área de círculos, volume de esferas, trabalho num campo de forças, comprimento de arco, centro de massa etc.

O TFC surgiu após a descoberta do processo de diferenciação, seja sob a ótica de Newton ou de Leibniz, mas há evidentemente outros cientistas que influenciaram nessa descoberta, tais como Arquimedes, Kepler, Cavalieri, Fermat, Wallis, Isaac Barrow etc, para citar alguns. Nas palavras de E. Hairer e G. Wanner [19],

\footnotetext{
${ }^{5}$ Essa fórmula está escrita numa notação moderna, diferente daquela escrita no século XVII.

${ }^{6}$ Assim, é plausível a associação de integral indefinida como sendo a operação inversa da derivada.
} 
O passo decisivo veio quando Newton, Leibniz, e Joh. Bernoulli descobriram independentemente que integração é a operação inversa da diferenciação, reduzindo assim os esforços dos pesquisadores acima a algumas regras de diferenciação (Tradução dos autores) ([19], p. 107).

O TFC é demonstrado levando-se em conta a relação entre integrais e antiderivadas, e doravante, naquela época, esforços seriam canalizados na busca por funções que tivessem primitivas. Em cursos iniciais de Cálculo há um foco no estudo sobre integrais em que se tem em mente o TFC e a busca por primitivas. Há também, às vezes, uma forte relação entre o conceito de integrais e o conceito de antiderivadas. Essa estratégia pedagógica não está em desacordo com o contexto histórico, mas deve-se cuidar, tal como também ocorreu na história da matemática, ao investigar integrais que contenham funções com pontos de singularidades, tal como $\int_{-2}^{3} \frac{1}{x-1} d x$, ou mesmo para integrais do tipo $\int_{1}^{2} e^{-x^{2}} d x$, em que $f(x)=e^{-x^{2}}$ não tem primitivas. Ou, ainda, como exposto no livro A Introduction to Analysis and Integration Theory, [28], em que se diz que o uso indiscriminado da integral definida de Newton-Leibniz pode gerar problemas de difícil explicação, tais como:

Era difícil explicar o que significava $\int_{-1}^{1} x^{-1} d x=\ln (-1)$; o lado esquerdo aparentava ser um número real, enquanto que o direito era imaginário (...) (Tradução dos autores) ([28], pviii).

Antes disso, em seu livro Philosophiae Naturalis Principia Mathematica, de 1687, Newton preocupa-se com a busca de áreas via quadraturas, ou seja, se o objetivo é determinar a área $A$ de uma região $R$, então busca-se por um quadrado cuja área seja igual a $A$. Já para Leibniz, o problema de encontrar áreas resumia-se a trabalhar com infinitésimos.

Com a definição acima de integral, a classe de funções integráveis, no sentido de Newton-Leibniz, será aquela para a qual existem primitivas. Incluídas nesta classe estão as funções polinomiais, trigonométricas etc., que representam, normalmente, a grande parte das funções tratadas em cursos de Cálculo. No entanto, uma grande parte de funções não são integráveis, no sentido de NewtonLeibniz. As funções do tipo escada - ou seja, uma função $f:[a, b] \rightarrow \mathbb{R}$ de tal forma que $[a, b]$ é particionado em $n$ intervalos disjuntos $I_{1}, I_{2}, \ldots, I_{n}$ tal que $f(x)=c_{k}$, para todo $x \in I_{k}$ com $k=1,2,3, \ldots, n-$ não são Newton-Leibniz integráveis.

A ideia de integral como antiderivada implica uma redução às derivadas do conceito de integrais. A expansão de funções como uma série de Taylor e a possibilidade de integração termo a termo ajuda a diminuir o impacto da não resolução de integrais devido à não existência de primitivas. No entanto, problemas associados a equação da onda e séries de Fourier "indicaram" que a descoberta de uma caracterização independente do conceito de integral passaria pela revisão do conceito de funções, e isso foi feito por A. Cauchy. A caracterização de integral baseada em Newton-Leibniz não possibilita, nos dias de hoje, a resolução de alguns problemas em áreas tais como estatística, teoria da informação, física, equações diferenciais etc., [18]. 


\subsection{Integrais de Cauchy}

O que significa "área de uma região"? A resposta a essa pergunta implica a definição precisa do conceito de integral, que até a época de A. Cauchy estava sendo caracterizado pelo processo inverso da derivada, ou seja, pelas integrais de Newton-Leibniz. As razões que levaram A. Cauchy a iniciar um processo de rigor na matemática e a introduzir o conceito de limites ainda constituem palco para muitas discussões. Para H. Lebesgue, Cauchy esboçou o rigor na matemática por motivos unicamente pedagógicos, devido aos seus cursos na École Polytechnique ([28] px).

Henri Lebesgue, por outro lado, acreditava que Cauchy tinha iniciado seu programa de rigor principalmente por razões pedagógicas, (...) (Tradução dos autores) ([28], px).

Com o objetivo de fornecer uma definição de integral independentemente do conceito de derivadas, Cauchy introduz o moderno conceito de integral, definido como limite de somas "infinitas". Em 1823, no Résumé des leçons donnés à l'École Royale Polytechnique sur le calcul infinitésimale, Cauchy define o conceito de integral definida para funções contínuas [10]. Em notação moderna tem-se a seguinte definição:

Definição 2. ([3], pp199-200) Se $I=[a, b]$ é um intervalo fechado limitado em $\mathbb{R}$, então uma partição de $I$ é um conjunto finito, ordenado, $\mathcal{P}=\left\{x_{0}, x_{1}, \ldots, x_{n-1}, x_{n}\right\}$ de pontos em $I$, tal que

$$
a=x_{0}<x_{1}<x_{2}<\ldots<x_{n}=b
$$

Os pontos de $\mathcal{P}$ são usados para dividir $I=[a, b]$ em subintervalos disjuntos

$$
I_{1}=\left[x_{0}, x_{1}\right] \quad I_{2}=\left[x_{1}, x_{2}\right], \ldots, I_{n}=\left[x_{n-1}, x_{n}\right] .
$$

Representa-se uma partição de $[a, b]$ por

$$
\mathcal{P}=\left\{\left[x_{i-1}, x_{i}\right]\right\}_{i=1}^{n} .
$$

Definição 3. Se $\mathcal{P}=\left\{\left[x_{i-1}, x_{i}\right]\right\}_{i=1}^{n}$ é uma partição de $[a, b]$, então a soma de Cauchy de uma função contínua $f:[a, b] \rightarrow \mathbb{R}$ correspondente a $\mathcal{P}$ é o número

$$
S(f ; \mathcal{P})=\sum_{i=1}^{n} f\left(x_{i-1}\right)\left(x_{i}-x_{i-1}\right) .
$$

Definição 4. Uma função contínua $f:[a, b] \rightarrow \mathbb{R}$ é dita ser Cauchy integrável em $[a, b]$ se existe um número $L \in \mathbb{R}$ tal que para cada $\varepsilon>0$ existe um $\delta>0$ tal que se $\mathcal{P}$ é uma partição de $[a, b]$ com $\|\mathcal{P}\|<\delta$, então

$$
|S(f ; \mathcal{P})-L|<\varepsilon
$$

O conjunto de todas as funções que são Cauchy integráveis em $[a, b]$ será denotado por $\mathcal{C}[a, b]$. Se $f \in \mathcal{C}[a, b]$, então o número $L$ é unicamente determinado e será chamado de integral de Cauchy de $f$ em $[a, b]$, comumente denotado por

$$
\mathcal{C} \int_{a}^{b} f(x) d x=L .
$$


Com base na noção de limites, Cauchy define integrais a partir de "somas infinitas." Nesses desenvolvimentos, Cauchy limita-se a abordar o conceito de integrais, via somas, independentemente de derivadas, apenas para funções contínuas,em um intervalo fechado e limitado $[a, b]$. É o início das definições das classes de funções integráveis. Com Cauchy, inicia-se um método construtivo para o cálculo de integrais, e a classe de funções integráveis, no sentido de Cauchy, torna-se independente do conceito de derivadas. Mas Cauchy não investigou apenas funções contínuas, ele estendeu seu conceito para funções descontínuas em um número finito de pontos de descontinuidade, funções com descontinuidades isoladas. Funções com pontos de descontinuidade não podiam ser integráveis, e métodos especiais precisavam ser elaborados para integrá-las. Para as funções do tipo $\frac{1}{(x-1)^{2}}$, Cauchy utiliza limites para sua integração e trabalha com integrais impróprias.

Definição 5. ([35], p505) Seja $f$ uma função contínua em um intervalo $(a, b]$ que é ilimitada em cada intervalo $(a, a+\delta)$. Então

$$
\int_{a}^{b} f(x) d x=\lim _{\delta \rightarrow 0^{+}} \int_{a+\delta}^{b} f(x) d x
$$

se esse limite existir. No caso de o limite existir a integral é dita ser convergente, caso contrário é dita ser divergente.

Com o fortalecimento do conceito de funções, fortalece-se também o conceito de integrais, e, daí, busca-se por novos desenvolvimentos do conceito de integral com vistas a aumentar a classe de funções integráveis. É o que ocorre com G. Riemann, que fornece um tratamento sistemático para as integrais, mais amplo que o de Cauchy.

\subsection{Integrais de Riemann}

O que é uma função integrável? Questionamento como esse foi elaborado por Riemann, quando propôs investigar com precisão o conceito de integral. Em 1854 ele questiona em seu artigo [31]:

Nossa primeira questão é portanto: que significado devemos dar para $\int_{a}^{b} f(x) d x$ ? (Tradução dos autores) (Riemann 1854, Werke, p239) ([19] p221).

O esclarecimento dessas questões leva ao entendimento e discernimento do que seja uma função integrável. Pode-se dizer que Riemann precisou rever o conceito de integrais, e de funções, para poder investigar os problemas deixados por Fourier. Riemann foi o primeiro a desenvolver um processo sistemático para avaliar condições de integrabilidade sobre funções. As somas de Cauchy consideram, em cada subintervalo de uma partição, o valor da esquerda, $x_{i-1}$, do subintervalo $\left[x_{i-1}, x_{i}\right]$ como argumento da função $f$. Riemann, por sua vez, aprofunda os estudos sobre a classe de funções integráveis e, ao invés de escolher, em cada partição o valor $x_{i-1}$, escolhe um valor qualquer, ou seja, considera um valor $t_{i} \in\left[x_{i-1}, x_{i}\right]$.

$$
\text { Cauchy: } \sum_{i=1}^{n} f\left(x_{i-1}\right)\left(x_{i}-x_{i-1}\right) \quad \text { Riemann: } \sum_{i=1}^{n} f\left(t_{i}\right)\left(x_{i}-x_{i-1}\right) \text {. }
$$


Essa flexibilidade permite estabelecer condições necessárias e suficientes para a existência da integral. Com isso, Riemann investiga as questões deixadas por J. P. Lejeune Dirichlet (1805-1859) e trabalha para responder à seguinte pergunta: Em quais casos uma função é integrável? Por isso atribui-se a Riemann o início das investigações sobre as condições de integrabilidade. No entanto, alguns conceitos básicos de análise (como limites, continuidade, continuidade uniforme, convergência uniforme etc.) ainda não eram conhecidos na época de Riemann e só viriam a ser entendidos por volta dos anos 1872 (final do século XIX). Também, mesmo sabendo que série de Fourier foi um dos pontos de investigação de Riemann, o qual resultou nos estudos sobre condições de integrabilidade, foi a aceitação de uma caracterização geral do conceito de funções que alavancou todo o processo.

G. Riemann definiu a integral definida de uma função $f$ de números reais [31], no intervalo fechado $[a, b]$, da seguinte maneira, em notação atual ([3], pp199-201):

Definição 6. ([3], p200) Seja $\mathcal{P}=\left\{\left[x_{i-1}, x_{i}\right]\right\}_{i=1}^{n}$ uma partição $^{7}$ de $I$. Uma etiqueta é um ponto $t_{i} \in I_{i}=\left[x_{i-1}, x_{i}\right]$, para $i=1,2, \ldots, n$. Uma partição etiquetada é um conjunto de pares ordenados

$$
\dot{\mathcal{P}}=\left\{\left(\left[x_{i-1}, x_{i}\right], t_{i}\right)\right\}_{i=1}^{n} .
$$

Definição 7. ([3], p200) Se $\dot{\mathcal{P}}=\left\{\left(\left[x_{i-1}, x_{i}\right], t_{i}\right)\right\}_{i=1}^{n}$ é uma partição etiquetada, então a soma de Riemann de uma função $f:[a, b] \rightarrow \mathbb{R}$ correspondente a $\dot{\mathcal{P}}$ é o número

$$
S(f ; \dot{\mathcal{P}})=\sum_{i=1}^{n} f\left(t_{i}\right)\left(x_{i}-x_{i-1}\right)
$$

Definição 8. ([3], p201) Uma função $f:[a, b] \rightarrow \mathbb{R}$ é dita ser Riemann integrável em $[a, b]$ se existe um número $L \in \mathbb{R}$ tal que para cada $\varepsilon>0$ existe um $\delta>0$ tal que se $\dot{\mathcal{P}}$ é uma partição etiquetada de $[a, b] \operatorname{com}\|\dot{\mathcal{P}}\|<\delta_{\varepsilon}$, então

$$
|S(f ; \dot{\mathcal{P}})-L|<\varepsilon .
$$

O conjunto de todas as funções que são Riemann integráveis em $[a, b]$ será denotado por $\mathcal{R}[a, b]$. Se $f \in \mathcal{R}[a, b]$, então o número $L$ é unicamente determinado e será chamado de integral de Riemann de $f$ em $[a, b]$, comumente denotado por

$$
\mathcal{R} \int_{a}^{b} f(x) d x=L
$$

As somas $S(f ; \dot{\mathcal{P}})$ são as famosas somas de Riemann, já em notação moderna [3]. No entanto, é comum observar a denominação "somas de Riemann" sendo aplicada a quaisquer tipo de somas cujo objetivo é calcular o valor de uma integral via inserção ou circunscrição de retângulos na região que se pretende calcular a área. Tal como em Cauchy, Riemann utilizou também de integrais impróprias. Num sentido estrito, integrais impróprias não podem ser ditas serem integrais de Riemann, pois lidam com funções ilimitadas (que não são Riemann integráveis) ([5], p30). Mas com as integrais impróprias, a classe de funções integráveis expande-se. O exemplo

$$
\int_{-1}^{1} \frac{1}{\sqrt{|x|}} d x
$$

${ }^{7}$ Leva-se em conta a definição de partição exposta na seção sobre Integrais de Cauchy. 
mostra uma função, $\frac{1}{\sqrt{|x|}}$, que é integrável (imprópria) no sentido de Riemann, mas

$$
\int_{-1}^{1} \frac{1}{x} d x
$$

não é uma integral resolvida no sentido de Riemann (integral imprópria). Isso mostra que a existência de antiderivadas (neste caso, $F(x)=\ln (|x|)$ ) não garante a existência de integrais impróprias ([5], pp30-31). Como será visto em exemplos nas seções que se seguem, há também funções que são integráveis no sentido de Riemann (integral imprópria) mas não o são no sentido de Lebesgue.

\section{Integrais de Lebesgue}

É aceito, na comunidade acadêmica, que as integrais de Newton e Leibniz eram insuficientes para impor desenvolvimentos na matemática, haja vista sua dependência ao conceito de derivadas. Além disso, funções como a função sinal $f(x)=\operatorname{sign}(x)$ não seriam integráveis em $[-1,1]$, por exemplo. No entanto a área da região entre o gráfico dessa função e o eixo $x$ nesse intervalo é 2, por isso Cauchy aborda integrais para funções contínuas e descontínuas em um número finito de pontos. Riemann segue esses passos e vai além. Caracteriza o que é "área sob uma curva" e integra funções com um número infinito de pontos de descontinuidade. Mesmo assim, o conceito de integral ainda se mantém sob a tutela do conceito de continuidade. "Quanto" de descontinuidade pode haver em uma função para que ela ainda seja integrável? A função de Dirichlet não é Riemann integrável, mas a função de Thomae é Riemann integrável ([1], p193), e ambas têm infinitos pontos de descontinuidade 8 . A resposta a essa pergunta foi dada por Lebesgue no trabalho Sur une généralisation de l'intégrale définie em 1901 e em sua tese de doutorado Intégrale, Longuer, Aire em 1902.

Na sequência, para fins de comparação entre as várias definições do conceito de integral, expõe-se o conceito de integral de Lebesgue de modo semelhante ao de Riemann, em notação moderna, tal como exposto em [39].

Definição 9. ([39], p19) Uma medida exterior $m^{*}(G)$ de um conjunto aberto $G \subset[0,1]$ é definida como o número real $\sum_{i}\left(b_{i}-a_{i}\right)$, em que $G=\cup_{i}\left(a_{i}, b_{i}\right)^{9}$.

Definição 10. ([39], p19) Uma medida exterior $m^{*}(A)$ de qualquer conjunto $A \subset[0,1]$ é definida como sendo o número real

$$
\sup \left\{m^{*}(G) \mid A \subset G \text { e } G \text { é um conjunto aberto em }[0,1]\right\} .
$$

Definição 11. ([39], p22) Uma medida interior $m_{*}(A)$ de qualquer conjunto $A \subset[0,1]$ é definida como sendo um número real $1-m^{*}([0,1] A)$.

\footnotetext{
${ }^{8}$ A função de Dirichlet tem um conjunto não enumerável de pontos de descontinuidade e a função de Thomae tem um conjunto enumerável de pontos de descontinuidade ([3], pp127-128).

${ }^{9}$ Leva-se em conta que cada conjunto não vazio aberto $G \subset \mathbb{R}$ pode ser expresso unicamente como uma união finita ou uma união infinita contável de pares disjuntos de intervalos abertos.
} 
Definição 12. ([39], p22) Um conjunto $A \subset[0,1]$ é dito ser Lebesgue mensurável se $m_{*}(A)=$ $m^{*}(A)$. Neste caso, a medida de $A$, denotada por $m(A)$, é o número $m_{*}(A)=m^{*}(A)$.

Definição 13. ([39], p47) Seja $A$ um subconjunto mensurável limitado de $\mathbb{R}$. Então $f: A \rightarrow \mathbb{R}$ é uma função mensurável em $A$ se $\{x \in A \mid c<f(x)\}$ é mensurável para cada número real $c$.

Definição 14. ([39], pp59-60) Se $f$ é uma função mensurável em um conjunto mensurável $A \subset \mathbb{R}$, se $P=\left(y_{0}, y_{1}, \ldots, y_{n}\right)$ é uma partição de

$$
[\ell, u]=[\sup \{f(x) \mid x \in A\}, 1+\inf \{f(x) \mid x \in A\}],
$$

e se

$$
y_{i}^{*} \in\left[y_{i-1}, y_{i}\right] \text { para } i=1,2, \ldots, n,
$$

então denomina-se

$$
L(f, P)=\sum_{i=1}^{n} y_{i}^{*} \cdot m\left(\left\{x \in A \mid y_{i-1} \leq f(x)<y_{i}\right\}\right)
$$

de soma de Lebesgue de $f$ relativa a $P$.

Definição 15. ([39], p60) Uma função mensurável $f: A \rightarrow \mathbb{R}$ é uma função Lebesgue integrável em $A$ se existe um número real $L$ tal que para cada $\varepsilon>0$, existe um $\delta>0$ tal que, se $\|P\|<\delta$, e se $L(f, P)$ é uma soma de Lebesgue de $f$ relativa a $P$, então $|L(f, P)-L|<\varepsilon$.

O número $L$, único, é chamado de integral de Lebesgue de $f$ em $A$, e denotado por

$$
\int_{A} f d m .
$$

Lebesgue elaborou uma "teoria da medida" para que seu novo conceito de integral pudesse ser desenvolvido. Mas a noção de medida deveria satisfazer a intuição comum, tal como na medida de um intervalo em $\mathbb{R}$. Por exemplo, ao se medir, com base na intuição, um intervalo $X=[a, b]$ e um intervalo $Y=(b, c]$, disjuntos e tal que $[a, b] \cup(b, c]=[a, c]=Z$, deve-se ter que a medida de $X$ mais a medida de $Y$ deve fornecer a medida de $Z$. Ou ainda, como mais um exemplo, estima-se que a medida de um subconjunto de $X$ não exceda a medida do próprio conjunto $X$. Além dessas, outras noções intuitivas sobre o conceito de medida deveriam ser satisfeitas. Mas uma delas foi muito significativa, qual seja, a ideia de que "o todo é a soma das partes." A generalização dessa ideia é conhecida como aditividade contável, ([8], pp86-90) ${ }^{10}$. Mas há recursos matemáticos que auxiliam na definição de medida, tal como uma $\sigma$-álgebra ${ }^{11}$, que é fechada para as operações usuais da teoria de conjuntos. A $\sigma$-álgebra gerada pela coleção de todos os intervalos abertos de $\mathbb{R}$ é chamada de $\sigma$-álgebra $\mathcal{B}$ de Émile Borel (1871-1956). Com base nisso, tem-se que cada conjunto de Borel de números reais é um conjunto Lebesgue mensurável ([7], p.112-113). Se $\mathcal{M}$ é a coleção de todos os conjuntos mensuráveis de $\mathbb{R}$, então existem conjuntos em $\mathcal{M}$ que não estão em $\mathcal{B}([2]$, p.311). Isso indica que há uma hierarquia de conjuntos: mensuráveis, de Borel, $\sigma$-álgebra,

\footnotetext{
${ }^{10}$ Há uma explicação muito interessante desse conceito no capítulo 3 de [7], bem como a explicação do complexo desenvolvimento da teoria da medida de Lebesgue.

${ }^{11}$ Uma coleção $S$ de subconjuntos de $E$ é chamada de classe contavelmente aditiva de conjuntos (ou $\sigma$-álgebra) se: i) $E \in S$, ii) se $A \in S$ então $(E-A) \in S$ e iii) se $\left\{A_{i}\right\}_{i=1}^{\infty} \subset S$ então $\cup_{i=1}^{\infty} A_{i} \in S$ ([39], p33).
} 
que satisfazem aditividade contável etc., os quais indicam também a classe de funções integráveis no sentido de Lebesgue ([7], p.114). Nota-se com isso o quanto que a noção de medida influencia no conceito de integral de Lebesgue.

Nota-se que Riemann aprimorou as somas de Cauchy, e Lebesgue, de modo análogo, aprimorou as somas de Riemann. Em vez de tomar as partições no domínio da função $f(x)$, como em Riemann, Lebesgue tomou as partições na imagem da função $f(x)$, mas para isso precisou medir os subconjuntos de $\mathbb{R}$.

\section{Riemann : Partições no domínio $(a, b)$}

Lebesgue : Partições na imagem $(f, \bar{f})$

O entendimento do conceito de continuidade revela o quanto "extensivo" é o conceito de integração de Riemann. Seja, por exemplo, para $x \in[0,1]$ e $\left\{r_{1}, r_{2}, \ldots, r_{n}, \ldots\right\}$ uma enumeração de todos os números racionais ([1], p210-211). Seja a função $g_{n}$ de números reais definida como segue. Seja $g_{1}(x)=1$ se $x=r_{1}$ e seja $g_{1}(x)=0$ caso contrário. Seja agora, $g_{2}(x)=1$ se $x$ é $r_{1}$ ou $r_{2}$, exclusivamente, e seja $g_{2}(x)=0$ em todos os outros pontos de $[0,1]$. Para cada $n \in \mathbb{N}$, seja

$$
g_{n}(x)= \begin{cases}1 & \text { se } x \in\left\{r_{1}, r_{2}, r_{3}, \ldots, r_{n}\right\} \\ 0 & \text { caso contrário. }\end{cases}
$$

Cada $g_{n}$ tem somente um número finito de descontinuidades, e portanto é Riemann integrável com

$$
\mathcal{R} \int_{0}^{1} g_{n}=0 .
$$

Mas a sequência de funções $\left(g_{n}\right)$ converge para a função $g(x)$ de Dirichlet, ponto a ponto, no intervalo $[0,1]$. Assim

$$
\lim _{n \rightarrow \infty} \mathcal{R} \int_{0}^{1} g_{n} \neq \mathcal{R} \int_{0}^{1} g
$$

Essa última equação seria válida se a convergência $g_{n}$ para $g$ fosse uma convergência uniforme ${ }^{12}$. Henri Lebesgue alterou a definição de integral de modo que a equação acima pudesse ser verdadeira. Para isso, Lebesgue introduz a ideia de medida de um conjunto e, com base nisso,

$$
\lim _{n \rightarrow \infty} \mathcal{L} \int_{0}^{1} g_{n}=\mathcal{L} \int_{0}^{1} g,
$$

e a função de Dirichlet torna-se Lebesgue Integrável. Há também deficiências nas integrais de Lebesgue. Nem todas as funções integráveis no sentido de Lebesgue satisfazem o TFC, e há funções cuja integral imprópria existe no sentido de Riemann, mas não no sentido de Lebesgue ([1], p212). Isso sugere novos desenvolvimentos no conceito de integrais. Isso foi o que O. Perron, A. Denjoy, J. Kurzweil e R. Henstock fizeram.

\footnotetext{
${ }^{12}$ Sobre esses conceitos ver [3], páginas 241 a 246.
} 


\section{Integrais de Kurzweil-Henstock}

Com base na teoria da medida e integração de Lebesgue, é possível dizer, acerca das integrais de Riemann, que uma função limitada é Riemann integrável se seu conjunto de pontos de descontinuidade for um conjunto de medida zero ([3], p.220). No entanto, mesmo na teoria de Lebesgue, ainda havia deficiências na teoria de integrais, indicando que alterações e ajustes faziam-se necessários. Com as integrais de Lebesgue tornou-se possível integrar a função de Dirichlet, mas ainda não era possível integrar todas as funções primitiváveis. Além disso, existem funções cuja integral imprópria existe e não são funções Lebesgue integráveis. Essas duas deficiências das integrais de Lebesgue, se assim se pode dizer, foram resolvidas também com as integrais de Kurzweil-Henstock. Em 1957 o matemático tcheco Jaroslav Kurzweil (1926-) em trabalhos de pesquisa em equações diferenciais fornece uma definição de integral equivalente à de Perron [26]. Mais tarde, o matemático britânico Ralph Henstock (1923-2007) desenvolve, independentemente, um conceito de integral equivalente ao de Kurzweil [22]. Em ambas as apresentações, o conceito fundamenta-se nas ideias simples das integrais de Riemann. Além disso, o conceito é amplo o suficiente para considerar as integrais de Riemann e de Lebesgue como casos particulares.

Definição 16. ([3], p149) Uma função calibre em um intervalo $I=[a, b]$ é uma função estritamente positiva definida em $I$. Se $\delta$ é um calibre em $I$, então uma partição etiquetada $\dot{\mathcal{P}}$ é dita ser $\delta$-fina se

$$
t_{i} \in I_{i} \subseteq\left[t_{i}-\delta\left(t_{i}\right), t_{i}+\delta\left(t_{i}\right)\right] \text { para } i=1,2, \ldots, n .
$$

Lema 1 (Cousin). ([3], p151) Se $\delta$ é um calibre definido no intervalo $[a, b]$, então existe uma partição $\delta$-fina de $[a, b]$.

Um ponto importante é a relação entre as integrais de Kurzweil-Henstock e o lema de Cousin, que é equivalente a um axioma de completude [32]. Pierre Cousin (1867-1933) provou, [12], que, se $I=[a, b] \subset \mathbb{R}$ é um intervalo não degenerado e se $\delta$ é qualquer calibre definido sobre $I$, então sempre existirá uma partição etiquetada de $I$, que é $\delta$-fina ([2], p11), base para a definição das integrais de Kurzweil-Henstock.

Definição 17. ([3], p290) ${ }^{13}$ Uma função $f:[a, b] \rightarrow \mathbb{R}$ é dita ser Kurzweil-Henstock integrável em $[a, b]$ se existe um número $L \in \mathbb{R}$ tal que para cada $\epsilon>0$ existe um calibre $\delta_{\varepsilon}>0$ em $[a, b]$ tal que se $\dot{\mathcal{P}}$ é qualquer partição $\delta_{\varepsilon}$-fina de $[a, b]$, então

$$
|S(f ; \dot{\mathcal{P}})-L|<\epsilon .
$$

O conjunto de todas as funções que são Kurzweil-Henstock integráveis em $[a, b]$ será denotado por $\mathcal{K} \mathcal{H}[a, b]$. Se $f \in \mathcal{K} \mathcal{H}[a, b]$, então o número $L$ é unicamente determinado e será chamado de integral de Kurzweil-Henstock de $f$ em $[a, b]$, comumente denotado por

$$
L=\mathcal{K} \mathcal{H} \int_{a}^{b} f(x) d x .
$$

Do mesmo modo que, com simples alterações na definição de integrais de Cauchy, Riemann expandiu sua classe de funções integráveis, as integrais de Kurzweil-Henstock foram expandidas graças

\footnotetext{
${ }^{13}$ No livro Introduction to Real Analysis, ([3], p290), os autores utilizam a denominação Integrais de Riemann
} generalizadas, em vez de integrais de Kurzweil-Henstock como denotadas nesse texto. 
também a simples alterações nas integrais de Riemann. Em Riemann considera-se um valor $\delta>0$, já em Kurzweil-Henstock considera-se uma função $\delta$ de valores estritamente positivos.

$$
\text { Riemann }: I_{i} \subseteq\left[t_{i}-\delta, t_{i}+\delta\right] \quad \text { Kurzweil-Henstock }: I_{i} \subseteq\left[t_{i}-\delta\left(t_{i}\right), t_{i}+\delta\left(t_{i}\right)\right]
$$

Observa-se que há vantagens e desvantagens nessas classes de funções integráveis expostas neste texto. Por exemplo, existem funções $f$ que são Kurzweil-Henstock integráveis mas o mesmo não ocorre com o valor absoluto dessas mesmas funções, ou seja, $f \in \mathcal{K} \mathcal{H}[a, b]$, mas $|f| \notin \mathcal{K} \mathcal{H}[a, b]$. Já, com as funções Riemann integráveis, se tem que se $f \in \mathcal{R}[a, b]$ então $|f| \in \mathcal{R}[a, b]([3]$, p. 304). Por outro lado, dentre as funções que pertencem à classe de funções que são Kurzweil-Henstock integráveis, torna-se possível identificar quais delas são Lebesgue integráveis. Para isso basta levar em conta a seguinte asserção: uma função $f \in \mathcal{L}[a, b]$ se, e somente se, ambas as funções, $f \in \mathcal{K} \mathcal{H}[a, b]$ e seu valor absoluto $|f| \in \mathcal{K} \mathcal{H}[a, b]([2]$, p.17).

\section{Exemplos}

Nessa seção apresentam-se exemplos de funções que são e que não são integráveis, nas abordagens expostas em seções anteriores. As demonstrações podem ser encontradas nas respectivas referências bibliográficas. Além de expor exemplos clássicos e facilitadores, no sentido didático, argumenta-se também que o assunto não se esgotou em termos de desenvolvimento científico. A classe de funções integráveis no sentido de Kurzweil-Henstock não aborda uma classe tão ampla como se imagina. Além disso sempre há prós e contras com relação às facilidades que essas integrais produzem no fazer matemático. Isso indica que novos desenvolvimentos ainda podem ser elaborados com o objetivo de ampliar ainda mais a classe de funções integráveis. No livro A Modern Theory of Integration, Robert Bartle escreve que ainda há desenvolvimentos a serem realizados na teoria da integração:

\section{(...) é surpreendente que novos desenvolvimentos continuem a surgir nessa teoria (...) (Tradução dos autores) ([2], pix).}

Exemplo 1. ([3], p206) Seja a função de Thomae $h:[0,1] \rightarrow \mathbb{R}$, com $h(x)=0$ se $x \in[0,1]$ é um número irracional; $h(0)=1$; e $h(x)=\frac{1}{n}$ se $x \in[0,1]$ é um número racional $x=\frac{m}{n}$ onde $m, n \in \mathbb{N}$ são primos entre si. A função de Thomae, que é contínua em cada número irracional e descontínua em cada número racional, é Riemann integrável em [0,1].

Exemplo 2. As funções $e^{-x^{2}}$, sen $x^{2}, \frac{1}{\ln x}, \frac{\operatorname{sen} x}{x}, \frac{\cos x}{x}$ e $\frac{x}{|x|}$ não são Newton-Leibniz integráveis.

Exemplo 3. ([3], p218) Seja $F(x)=x^{2} \cos \left(\frac{1}{x^{2}}\right)$ para $x \in(0,1]$ e $F(0)=0$. A função

$$
F^{\prime}(x)=2 x \cos \frac{1}{x^{2}}+\frac{2}{x} \operatorname{sen}\left(\frac{1}{x^{2}}\right),
$$

para $x \in(0,1]$ com $F^{\prime}(0)=0$ não é limitada em $[0,1]$ e $F(x)$ é contínua e diferenciável em cada ponto em $[0,1]$. $F^{\prime}$ é uma função que não é Riemann integrável, é Newton-Leibniz integrável e é Kurzweil-Henstock integrável em $[0,1]$. 
Exemplo 4. A função $f(x)$ de Dirichlet,

$$
f(x)=\left\{\begin{array}{lll}
1 & \text { se } & x \in \mathbb{Q} \\
0 & \text { se } & x \in \mathbb{Q}
\end{array}\right.
$$

introduzida por P. G. L. Dirichlet em 1829, é Kurzweil-Henstock integrável em [0,1], ([3], p291), é Lebesgue integrável em [0,1], ([8], p21), mas não é Riemann integrável ([3], p209) e nem NewtonLeibniz integrável

Exemplo 5. ([40], p71) A função

$$
f(x)=\frac{1}{x}
$$

para $x \neq 0$ não é Kurzweil-Henstock integrável em $[0,1]$. Também, $f(x)=\frac{1}{x} \operatorname{com} x \in[1,+\infty)$ não é Kurzweil-Henstock integrável em $[1,+\infty)$ ([3], p314).

Exemplo 6. ([3], p288 e p311) Na integral imprópria de Dirichlet,

$$
\int_{0}^{\infty} \frac{\operatorname{sen} x}{x} d x
$$

a função $\frac{\operatorname{sen} x}{x}$ pertence a $\mathcal{K} \mathcal{H}[0, \infty)$, mas não é Lebesgue integrável em $[0, \infty)$. Nota-se também que na integral com valor absoluto $\int_{0}^{\infty}\left|\frac{\operatorname{sen} x}{x}\right| d x$, a função $\left|\frac{\operatorname{sen} x}{x}\right|$ não é nem Kurzweil-Henstock, nem Lebesgue integrável em $[0, \infty)$.

Exemplo 7. ([40], p18) ([5], p91) A função

$$
f(x)=2 x \operatorname{sen}\left(\frac{1}{x}\right)-\cos \left(\frac{1}{x}\right),
$$

é descontínua e limitada, para $x \neq 0, \operatorname{com} f(0)=0$. A função $F(x)=x^{2} \operatorname{sen}\left(\frac{1}{x}\right), \operatorname{com} F(0)=0$ é tal que $F^{\prime}=f$. Assim, $f$ é Riemann integrável e também é Newton-Leibniz integrável em [0,1].

Exemplo 8. ([3], p215, ex. 12), ([40], p. 18) A função

$$
f(x)=\operatorname{sen}\left(\frac{1}{x}\right)
$$

para $x \in(0,1]$ e $f(0)=0$ é Riemann integrável, mas não é Newton-Leibniz integrável.

Exemplo 9. ([4], p254), ([3], p302) A função ilimitada

$$
f(x)=\frac{1}{\sqrt{x}}
$$

com $f(0)=0$ é Newton-Leibniz integrável, é Cauchy integrável, é Riemann integrável em $(0,1]$ e é também Kurzweil-Henstock integrável em [0,1]. A função $g(x)=f(x)$. sen $\left(\frac{1}{x}\right)$ para $x \in(0,1] \mathrm{com}$ $g(0)=0$ é Lebesgue integrável em [0,1] ([3], p307). 
Exemplo 10. ([5] pp33-35) A função

$$
f(x)=\sum_{n=1}^{\infty} \frac{((n x))}{n^{2}}
$$

é Riemann integrável, mas é descontínua em todos os números racionais. Neste caso, a função $((x))$ é definida como:

$$
((x))=\left\{\begin{array}{lll}
x-\lfloor x\rfloor & \text { se } \quad\lfloor x\rfloor \leq x<\lfloor x\rfloor+\frac{1}{2} \\
0 & \text { se } x=\lfloor x\rfloor+\frac{1}{2} \\
x-\lfloor x\rfloor-1 & \text { se }\lfloor x\rfloor+\frac{1}{2}<x<\lfloor x\rfloor+1
\end{array}\right.
$$

Exemplo 11. ([3], p301) Seja $F(x)=x \cos \left(\frac{\pi}{x}\right)$ para $x \in(0,1] \operatorname{com} F(0)=0$. A função $f(x)=$ $F^{\prime}(x)=\frac{\pi}{x} \operatorname{sen}\left(\frac{\pi}{x}\right)+\cos \left(\frac{\pi}{x}\right), \operatorname{com} f(0)=F^{\prime}(0)=0$, é Kurzweil-Henstock integrável em [0,1], mas $|f(x)|=\left|F^{\prime}(x)\right| \notin \mathcal{K} \mathcal{H}[0,1]$.

Exemplo 12. ([8], p194) A função $f(x)=2 x \operatorname{sen}\left(\frac{\pi}{x^{2}}\right)-\frac{2 \pi}{x} \cos \left(\frac{\pi}{x^{2}}\right)$ para $x \in(0,1]$ e $f(0)=0$ é Kurzweil-Henstock integrável, mas não é Lebesgue integrável.

Exemplo 13. ([5], p189) Seja $f(x)=2 x \operatorname{sen}\left(x^{-2}\right)-2 x^{-1} \cos \left(x^{-2}\right)$. A integral imprópria

$$
\int_{0}^{1} f(x) d x
$$

existe no sentido de Riemann, mas não existe no sentido de Lebesgue.

Exemplo 14. Seja a função $F(x)=x^{2} \operatorname{sen}\left(x^{-2}\right)$, para $x \neq 0$ e $F(0)=0$.

Seja $F^{\prime}(x)=2 x \operatorname{sen}\left(x^{-2}\right)-2 x^{-1} \cos \left(x^{-2}\right)$. $F^{\prime}$ é integrável no sentido de Newton-Leibniz e é Kurzweil-Henstock integrável em $[0,1]([40], \mathrm{p} 71)$, mas $F^{\prime}$ não é Riemann integrável ${ }^{14}$ ([5], p89).

Exemplo 15. ([3], p225) Se $h:[0,1] \rightarrow \mathbb{R}$ é a função de Thomae e se $\operatorname{sign}(x)$ é a função Sinal, então a função composta $\operatorname{sign}(x) \circ h$ não é Riemann integrável, mesmo que as funções de Thomae e Sinal sejam Riemann integráveis.

A partir do exposto nas seções anteriores, e nos exemplos desta seção, é possível exibir um quadro comparativo em que as funções integráveis, segundo sua definição particular, apresentam-se numa relação entre conjuntos.

\footnotetext{
${ }^{14}$ Não se leva em conta, neste caso, as integrais impróprias de Riemann.
} 


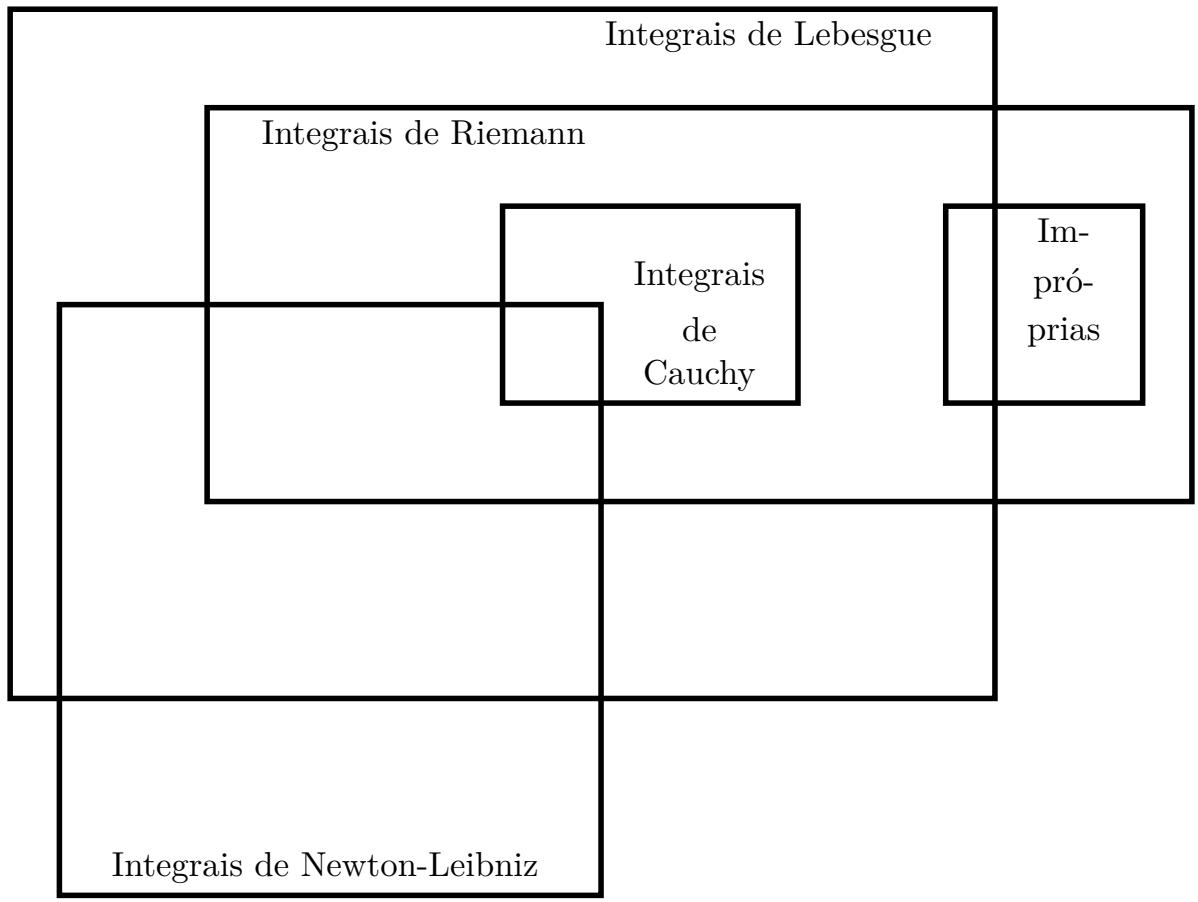

\section{Conclusão}

A exposição histórica com olhares na gênese do conhecimento, [36], torna-se fundamental como estratégia pedagógica, haja vista que esclarece os desenvolvimentos que são essenciais para a compreensão de conceitos matemáticos e para a percepção de que na matemática há processos de transformação e interação. Além disso, ajusta, destarte, os caminhos que formaram a construção de determinados conceitos. No caso do presente trabalho, o conceito de integrais é exposto e, tal como no quadro comparativo acima, são elencados exemplos, não exaustivos, onde a alteração (ou ajuste) do conceito de integrais impactou na expansão da classe de funções integráveis e na interação da matemática com outros campos do conhecimento. Urge que esclarecimentos, em cursos de Cálculo, sejam feitos em paralelo à apresentação de conceitos matemáticos. Caso não se explicite, num curso de Cálculo, por exemplo, qual é a abordagem de integrais adotada, dificuldades de leitura podem surgir, tal como no seguinte exemplo, sobre qual resposta pode ser considerada correta.

Exemplo 16. No escopo de integração via Riemann, a função de números reais $f(x)=\frac{1}{\sqrt{x}}$ defi- 
nida, por exemplo, num intervalo $(0,2]$, pode ser resolvida, num curso de Cálculo, com a aplicação das técnicas de integrais impróprias:

$$
\int_{0}^{2} \frac{1}{\sqrt{x}} d x=\lim _{a \rightarrow 0^{+}} \int_{a}^{2} \frac{1}{\sqrt{x}} d x=\lim _{a \rightarrow 0^{+}}[2 \sqrt{x}]_{a}^{2}=\lim _{a \rightarrow 0^{+}}[2 \sqrt{2}-2 \sqrt{a}]=2 \sqrt{2} .
$$

Mas, no escopo das integrais de Kurzweil-Henstock, essa mesma função pode ser resolvida de modo imediato:

$$
\int_{0}^{2} \frac{1}{\sqrt{x}} d x=[2 \sqrt{x}]_{0}^{2}=[2 \sqrt{2}-2 \sqrt{0}]=2 \sqrt{2}
$$

Vale citar um comentário de R. Bartle:

\section{Esses processos de limites não são necessários quando se trata de integrais de Riemann generalizadas. (Tradução dos autores) ([3], p302).}

Esse exemplo indica o quanto é importante o conhecimento de conceitos matemáticos e também da exibição de sua trajetória histórica e conceitual. Esses "caminhos" que formam a gênese de um conceito são vitais para se ter uma matemática conexa com seu desenvolvimento. É evidente que exemplos que desvelam o surgimento de um conceito existem em vários setores da matemática, haja vista que a evolução da matemática dá-se por processos dedutivos e indutivos, caracterizando uma ciência cumulativa. No entanto, como em toda ciência, há alterações e inovações em seus aspectos teóricos. O conceito de integrais é um desses exemplos. As facilidades de acesso às informações no mundo atual podem ser utilizadas para clarear e precisar os conceitos matemáticos. Desse modo, algumas confusões podem ser evitadas, tais como: utilizar somas de Riemann numa definição de integrais de Darboux [13], dizer de funções integráveis como sendo funções Newton-Leibniz integráveis; caracterizar somas de Cauchy como sendo somas de Riemann; dizer que a função $f(x)=\frac{1}{\sqrt{x}}$ não é integrável em $(0,2]$ sem dizer do conceito de integral no qual a função está inserida (exemplo 16); esquivar-se de uma caracterização de diferenças entre integrais construtivas e integrais descritivas etc. Outro cuidado que se deve ter refere-se ao Teorema Fundamental do Cálculo, pois esse pode induzir ao pensamento de que "ser integrável" é possuir primitivas. Atualmente se faz necessário que, ao se definir um conceito de integral, se explicite também a que tipo de integral se refere. O esclarecimento desse cenário, entende-se, é uma estratégia pedagógica com vistas às melhorias na compreensão de conceitos matemáticos em Cálculo.

\section{Agradecimentos}

Uma pequena parte deste artigo foi apresentada no $V$ Colóquio de Matemática da Região CentroOeste, ocorrido no Instituto Federal de Educação, Ciência e Tecnologia de Goiás, na cidade de Goiânia, de 26 a 30 de novembro de 2018, com auxílio financeiro, Projeto Faepex-Unicamp-3082/18 - Prof. J. C. Magossi. O presente trabalho foi realizado com apoio da Coordenação de Aperfeiçoamento de Pessoal de Nível Superior - Brasil (Capes) - Código de Financiamento 001. 


\section{Referências}

[1] Abbott, Stephen. Understanding Analysis. Springer, New York, 2001.

[2] Bartle, R. G. A Modern Theory of Integration. Graduate Studies in Mathematics, Volume 32. American Mathematical Society, Providence, Rhode Island, 2001.

[3] Bartle, R.G., Sherbert, D.R. Introduction to Real Analysis, Fourth Edition, Wiley India Edition, New Delhi, 2011.

[4] Bressoud, David M. A radical approach to real analysis. Vol. 2. Mathematical Association of America, 2007.

[5] Bressoud, David M. A radical approach to Lebesgue's theory of integration. Cambridge University Press, 2008.

[6] Bressoud, David M. Historical reflections on teaching the fundamental theorem of integral calculus. The American Mathematical Monthly, v. 118, n. 2, p. 99-115, 2011.

[7] Burk, Frank. Lebesgue Measure and Integration - An Introduction. John Wiley \& Sons, New York, 1998.

[8] Burk, Frank. A Garden of Integrals. Dolciani Mathematical Expositions, 31. The Mathematical Association of America-MAA Press. Washington DC, 2007.

[9] Cajori, Florian. A History of Mathematical Notations. Two Volumes Bound as One. I. Notations in Elementary Mathematics. II. Notations Mainly in Higher Mathematics. Dover Publications, New York, 1993.La Salle, Illinois: Open Court, 1928?29. 1993.

[10] Cauchy, Augustin-Louis. Résumé des leçons données à l'école royale polytechnique sur le calcul infinitésimal. Vol. 1. Imprimerie royale, 1823.

[11] Courant, R. , John, F.. Introduction to Calculus and Analysis I. Reprinted of the 1989 Edition. Springer-Verlag, Berlin. 1999.

[12] Cousin, Pierre. Sur les fonctions de $n$ variables complexes. Acta Matematica, 19, pp.1-62, 1895.

[13] Darboux, G. Mémoire sur la théorie des fonctions discontinues. Annales ENS (Vol. 2, No. 4, pp. 57-112), 1875.

[14] Loomis, Elisha Scott. The Pythagorean Proposition. Classics in Mathematics Education Series. 1968.

[15] Fourier, Joseph. Théorie analytique de la Chaleur, Firmin-Didot père et fils, Paris, 1822. Facsimile, Ed. Jacques Gabay, Paris, 1988.

[16] Gordon, R. A. The Integrals of Lebesgue, Denjoy, Perron, and Henstock. No. 4. American Mathematical Society, 1994.

[17] Grabiner, Judith V. The Origin of Cauchy's Rigorous Calculus. Dover Publications, Inc., New York, 1981.

[18] Grattan-Guinness, I. Joseph Fourier and the Revolution in Mathematical Physics. Journal of the Institute of Mathematics and its Applications, 5, 230-253, 1969.

[19] Hairer, E., Wanner, G. Analysis by Its History. New York, Springer-Verlag, 1996.

[20] Hawkins, T. Lebesgue's theory of integration. Its origins and development. 2nd edition, Chelsea Pub. Co., New York, 1975. 
[21] Henstock, R. Theory of integration. Butterworths, 1963.

[22] Henstock, Ralph. Definitions of Riemann type of the variational integrals. Proceedings of London Mathematical Society, (3), 11: 410-418, 1961.

[23] Hoare, G.T.Q., Lord, N. J. 'Intégrale, longueur, aire' the Centenary of the Lebesgue Integral. The Mathematical Gazette, Vol. 86, No. 505, pp. 3-27, Mar., 2002.

[24] Jahnke, H. N., A History of Analysis, History of Mathematics, Volume 24, American Mathematical Society, 2003.

[25] Kuhn, Thomas. A Estrutura das Revoluções Científicas. Editora Perspectiva, São Paulo, 1994.

[26] Kurzweil, J. Generalized ordinary differential equations and continuous dependence on a parameter. Czechoslovak Mathematical Journal, 7.3: 418-449, 1957.

[27] Mawhin, J. Analyse: fondements, techniques, évolution. De Boeck Université, Brussels, 1992. Second edition, 1997.

[28] Phillips, Esther R. A Introduction to Analysis and Integration Theory. Dover Publications, New York, 1984.

[29] Reid, Constance. Hilbert-Courant. Springer Science \& Business Media, 1986.

[30] Reid, Constance. Courant. Springer Science \& Business Media, 2013.

[31] Riemann, Bernhard. Ueber die Darstellbarkeit einer Function durch eine trigonometrische Reihe. Habilitation thesis, Göttingen, 1854, Werke, p. 227-271.

[32] Rioul, Olivier e Magossi, José Carlos (2018) A Local-Global Principle for the Real Continuum. Studia Logica, Vol. 47, Capítulo 11, pp. 213-240. Special edition of Trends in Logic 47: Contradictions, from Consistency to Inconsistency. Springer International Publishing, 2018.

[33] Rosentrater, C. R. Varieties of Integration. Dolciani Mathematical Expositions. The Mathematical Association of America-MAA Press. Washington DC, 2015.

[34] Schwartz, L. Theorie des Distributions. 3a, Ed. Herman, Paris, 1966.

[35] Thomson, Brian S., Judith B. Bruckner, and Andrew M. Bruckner. Elementary real analysis. CreateSpace, Second edition, 2008.

[36] Toeplitz, O. The Calculus - A Genetic Approach. Chicago University Press, Chicago, 2007.

[37] Van Vleck, E. B. The Influence of Fourier series on the development of mathematics. Science 39, 113-124, 1914.

[38] Volterra, Vito. Sui principii del calcolo integrale. Giornale di Matematiche Battaglini, 19, 333-372, 1881.

[39] Wilcox, H. J., Myers, D. L. An Introduction to Lebesgue Integration and Fourier Series. Dover Publications, New York, 1978.

[40] Yee, L. P., Výborný, R. The Integral: An Easy Approach after Kurzweil and Henstock.Cambridge University Press, Cambridge, 2000.

[41] Youschkevitch, A. P. The concept of function up to the middle of the 19th century. Archive for History of Exact Sciences. 16 (1): 37-85, 1976. 
José C. Magossi e Antônio C. C. Barros Faculdade de Tecnologia - FT - Unicamp <magossi@ft.unicamp.br> <a810088@dac.unicamp.br>

Recebido: 06/06/2019

Publicado: 20/12/2019 